\title{
A Class of Functions satisfying certain boundary value problems
}

\author{
Hemant Kumar ${ }^{1^{*}}$ \\ ${ }^{1}$ Department of Mathematics, D. A. V. Postgraduate College Kanpur-208001, Uttar Pradesh, India \\ *Corresponding author: palhemant2007@ rediffmail.com
}

Received: 20.5.2021; Revised: 21.8.2021; Accepted: 5.11.20021

(C) Society for Himalayan Action Research and Development

\begin{abstract}
In this paper for constructing of a class of functions consisting of integral representations, we introduce a double integral, a formula pertaining to extended fractal strings, consisting of separate variables functions in the integrand. Further by this double integral formula, we determine various functions, integrals and contour integral representations on introducing different special functions for these integrand functions connecting to RiemannLiouville and Weyl fractional integral functions. Finally, we apply our obtained results to find certain boundary value problems and present precise examples.
\end{abstract}

Key words: A double integral, A class of functions, Riemann-Liouville and Weyl fractional integrals, Contour integral representations, Boundary value problems.

2010 Mathematics Subject Classification: 26A33, 33E20, 33C60, 30E25.

\section{Introduction}

Lapidus and Frankenhuijsen $(2000,2006)$ have studied many approximations via fractal geometry and with the number theory as on application of complex dimensions and zeta functions. Again, Lapidus and Frankenhuijsen in (2006, p. 134) analyzed various explicit formulas for generalized fractal strings in an extension of a double integral given by

$$
\Psi(k)=\int_{0}^{\infty} \int_{y}^{\infty} \frac{(x-y)^{k-1}}{(k-1) !} \varphi(x) \psi(y) d x d y .
$$

In other words, the study of fractal geometry with zeta functions and number theory has explained by the double integral (1.1) for a test function $\varphi(x)$ and by $\mathrm{k}$ - th anti-derivative of $\psi(y)$, where the limits of the integration are $x=y, x=\infty, y=0$ and $y=\infty$, and $k=1,2,3, \ldots$. Here in this theory, a classical Dirac delta function (Lapidus and Frankenhuijsen 2006, p. 135) in form of contour integral representation is generalized into Heaviside function to discuss distinguished fractal geometry and zeta functions.

In recent investigation, we introduce a class of integral representations, $\Omega_{a}^{(k)}$, for $a \geq 0, k=1,2,3, \ldots$; concerning to a double integral given by

$$
\begin{aligned}
& \Psi_{a}^{(k)}=\int_{a}^{\infty} \int_{y}^{\infty} \frac{(x-y)^{k-1}}{(k-1) !} \varphi(x) \psi(y) d x d y, \quad \text { where, limits of the integration are } \\
& x=y, x=\infty, y=a \text { and } y=\infty ; k=1,2,3, \ldots, \text { defined as } \\
& \Omega_{a}^{(k)}=\left\{\Psi_{a}^{(k)}: \Psi_{a}^{(k)}=\int_{a}^{\infty} \int_{y}^{\infty} \frac{(x-y)^{k-1}}{(k-1) !} \varphi(x) \psi(y) d x d y<\infty\right\} .
\end{aligned}
$$


By change of order of integration in the double integral $\Psi_{a}^{(k)}$, given in Eqn. (1.2), we write it in the form of an integral representations

$$
\Psi_{a}^{(k)}=\int_{a}^{\infty} \varphi(x)\left\{\int_{a}^{x} \frac{(x-y)^{k-1}}{(k-1) !} \psi(y) d y\right\} d x<\infty \forall \varphi
$$

and $\psi$, real or complex.

Clearly, by the formulae (1.1) and (1.2) or (1.4), we have

$$
\lim _{a \rightarrow 0} \Psi_{a}^{(k)}=\Psi^{(k)}
$$

In Eqn. (1.4), the inner integral $\int_{a}^{x} \frac{(x-y)^{k-1}}{(k-1) !} \psi(y) d y$ is the anti-derivative of ${ }^{\psi}$, given by (Oldham and Spanier 2002 p. 27)

$$
\begin{aligned}
& \frac{d^{-k} \psi}{[d(x-a)]^{-k}}=\psi^{(-k)}(x)=\int_{a}^{x} \frac{(x-y)^{k-1}}{(k-1) !} \psi(y) d y \\
& \quad=\int_{a}^{x} d y_{k-1} \int_{a}^{y_{k-1}} d y_{k-2} \cdots \int_{a}^{y_{2}} d y_{1} \int_{a}^{y_{1}} \psi\left(y_{0}\right) d y_{0} .
\end{aligned}
$$

So that the integral (1.4) becomes interesting as

$\Psi_{a}^{(k)}=\int_{a}^{\infty} \varphi(x) \psi^{(-k)}(x) d x$.

Now, for further extensions and exploring above given ideas for $a \geq 0, \alpha, s \in \mathbb{C}$, where $\mathbb{C}$, a set of complex numbers and $\mathbb{R}^{+}$, a set of positive real numbers, for $\Re(\alpha), \Re(s) \in \mathbb{R}^{+}$, we consider a slight generalization of (1.2) as

$$
\Psi_{a}^{(\alpha)}(s)=\int_{a}^{\infty} \int_{y}^{\infty} \frac{(x-y)^{\alpha-1}}{\Gamma(\alpha)} \varphi(x s) \psi(y) d x d y<\infty, \text { and } \Psi_{a}^{(\alpha)}(s) \in \Omega_{a}^{(\alpha)} .
$$

In which, the class $\Omega_{a}^{(\alpha)}$ is defined by

$$
\Omega_{a}^{(\alpha)}=\left\{\Psi_{a}^{(\alpha)}: \Psi_{a}^{(k)}=\int_{a}^{\infty} \int_{y}^{\infty} \frac{(x-y)^{\alpha-1}}{\Gamma(\alpha)} \varphi(x s) \psi(y) d x d y<\infty, \forall \Re(\alpha), \Re(s) \in \mathbb{R}^{+}\right\} .
$$

Here, in the present researches via double integral (1.7) of class (1.8), we introduce many special functions for the separate variables functions of its integrand functions and then obtain various integral representations involving Riemann-Liouville integral and Weyl integral functions to this class. In this connection, we also evaluate definite contour integral representations via this double integral. Finally, we apply our obtained results to find certain boundary value problems and propose few examples.

1. Various functions via a double integral (1.7) of a class $\quad \Omega_{a}^{(\alpha)}(1.8)$ 
In this section, we derive various functions and integral representations connected to many special functions as on introducing various hypergeometric functions in place of the integrand functions and in the double integral (1.7) belonging to a class $\Omega_{a}^{(\alpha)}$, given in (1.8).

In view of the Riemann - Liouville fractional integral function given by (Diethelm 2010, Mathai and Haubold 2008)

$$
{ }_{a} I_{x}^{\alpha} \psi(x)=\int_{a}^{x} \frac{(x-t)^{\alpha-1}}{\Gamma(\alpha)} \psi(t) d t, \Re(\alpha)>0
$$

and on applying the techniques, to find the relation (1.4), we obtain the formula (1.7) in the form of an integral representations as

$$
\Psi_{a}^{(\alpha)}(s)=\int_{a}^{\infty} \varphi(x s)\left\{{ }_{a} I_{x}^{\alpha} \psi(x)\right\} d x<\infty, \Re(\alpha)>0, \Re(s)>0 .
$$

Here in the integral representation (2.2), $\Re(\alpha)>0, \Re(s)>0$ and $\forall x \in[a, \infty),\left\{{ }_{a} I_{x}^{\alpha} \psi(x)\right\} \in[a, \infty)$, there exists $\Psi_{a}^{(\alpha)}(s)<\infty$, thus $\Psi_{a}^{(\alpha)}(s) \in \Omega_{a}^{(\alpha)}$.

Now for evaluating of certain functions and integral representations of this class, we present following theorems as on introducing definite special functions in place of the functions and $\varphi$, given by:

Theorem 2.1. If $\Re(\alpha)>0, \Re(\beta)>0, \gamma \in \mathbb{C}$, consider $\psi(x)=\left[(x-a)^{\beta-1}(c x+d)^{\gamma}\right\rceil$ for $\left|\arg \left(-\frac{(a-x) c}{(a c+d)}\right)\right|<\pi$, where, and being constants, then by the representation (2.2), there exists an integral representation

$$
\Psi_{a}^{(\alpha)}(s)=(a c+d)^{\gamma} \frac{\Gamma(\beta)}{\Gamma(\alpha+\beta)} \int_{a}^{\infty}(x-a)^{\alpha+\beta-1}{ }_{2} F_{1}\left(\begin{array}{l}
\beta,-\gamma ; \\
\alpha+\beta ;
\end{array}-\frac{c(x-a)}{(a c+d)}\right) \varphi(x s) d x, \Re(s)>0 .
$$

Proof. For the conditions $\Re(\alpha)>0, \Re(\beta)>0, \gamma \in \mathbb{C}$, and $\left|\arg \left(\frac{(a-x) c}{(a c+d)}\right)\right|<\pi$, where, and being constants, set $\psi(x)=\left[(x-a)^{\beta-1}(c x+d)^{\gamma}\right\rceil$, in the formula (2.2), and then use the Riemann - Liouville $\psi$

fractional integral (2.1) for to apply the result due to (Mathai and Haubold 2008, p. 102, Example 2.4.3), given by

$$
{ }_{a} I_{x}^{\alpha}\left[(x-a)^{\beta-1}(c x+d)^{\gamma}\right]=(a c+d)^{\gamma}(x-a)^{\alpha+\beta-1} \frac{\Gamma(\beta)}{\Gamma(\alpha+\beta)}{ }_{2} F_{1}\left(\begin{array}{l}
\beta,-\gamma ; \\
\alpha+\beta ;
\end{array}-\frac{c(x-a)}{(a c+d)}\right) .
$$

Finally, by the formulae (2.2) and (2.4), we obtain the integral representation (2.3). 
Corollary 2.1. Again, if in the Theorem 2.1, under the condition $0<\Re(\alpha+\beta)<1$ and for the testing function $\varphi(x)=\cos x$, and then for $\Re(s)>0$, by the result of (Mathai and Haubold 2008, p. 105, Example 2.4.10), there exists a function

$$
\Psi_{a}^{(\alpha)}(s)=(a c+d)^{\gamma} \frac{\Gamma(\beta)}{\Gamma(\alpha+\beta)} \int_{a}^{\infty}(x-a)^{\alpha+\beta-1}{ }_{2} F_{1}\left(\begin{array}{l}
\beta,-\gamma ; \\
\alpha+\beta ;
\end{array}-\frac{c(x-a)}{(a c+d)}\right) \cos (x s) d x
$$

$$
\begin{aligned}
& =\frac{(a c+d)^{\gamma}}{s^{\alpha+\beta}} \frac{\Gamma(\beta)}{\Gamma(\alpha+\beta)} \sum_{k=0}^{\infty} \frac{(\beta)_{k}(-\gamma)_{k}}{(\alpha+\beta)_{k} k !} \Gamma(\alpha+\beta+k)\left(-\frac{c}{(a c+d) s}\right)^{k} \cos \left(a s+(\alpha+\beta+k) \frac{\pi}{2}\right) \\
& =\text { Real part of } \frac{(a c+d)^{\gamma}}{s^{a+\beta}} \Gamma(\beta) \exp \left[i\left\{a s+(\alpha+\beta) \frac{\pi}{2}\right\}\right] \sum_{k=0}^{\infty} \frac{(\beta)_{k}(-\gamma)_{k}}{k !}\left(-\frac{c}{(a c+d) s}\right)^{k}\left\{\exp \left[i \frac{\pi}{2}\right]\right\}^{k} \\
& \left.=\text { Real part of } \frac{(a c+d)^{\gamma}}{s^{a+\beta}} \Gamma(\beta) \exp \left[i\left\{a s+(\alpha+\beta) \frac{\pi}{2}\right\}\right]{ }_{2} F_{0}\left(\begin{array}{c}
\beta,-\gamma ; \\
-;
\end{array} \frac{c}{s(a c+d)}\right) \exp \left[i \frac{3 \pi}{2}\right]\right) \text {. }
\end{aligned}
$$

Corollary 2.2. Further, if in the Theorem 2.1, $0<\Re(\alpha+\beta)<1$ and the testing function $\varphi(x)=\sin x$, then for $\Re(s)>0$, use the result of (Mathai and Haubold 2008, p. 105, Example 2.4.11), there exists another function

$$
\Psi_{a}^{(\alpha)}(s)=(a c+d)^{\gamma} \frac{\Gamma(\beta)}{\Gamma(\alpha+\beta)} \int_{a}^{\infty}(x-a)^{\alpha+\beta-1}{ }_{2} F_{1}\left(\begin{array}{l}
\beta,-\gamma ; \\
\alpha+\beta ;
\end{array}-\frac{c(x-a)}{(a c+d)}\right) \sin (x s) d x
$$

$=\frac{(a c+d)^{\gamma}}{s^{a+\beta}} \Gamma(\beta) \sum_{k=0}^{\infty} \frac{(\beta)_{k}(-\gamma)_{k}}{k !}\left(-\frac{c}{(a c+d) s}\right)^{k} \sin \left(a s+(\alpha+\beta+k) \frac{\pi}{2}\right)$

$=$ Imaginary part of $\frac{(a c+d)^{\gamma}}{s^{\alpha+\beta}} \Gamma(\beta) \exp \left[i\left\{a s+(\alpha+\beta) \frac{\pi}{2}\right\}\right]{ }_{2} F_{0}\left(\begin{array}{c}\beta,-\gamma ; \\ -;\end{array}\left(\frac{c}{(a c+d) s}\right) \exp \left[i \frac{3 \pi}{2}\right]\right)$.

Theorem 2.2. For $\Re(\alpha)>0, \Re(\beta)>0, a \neq c$, consider $\psi(x)=\left[\frac{(x-a)^{\beta-1}}{(x \pm c)^{\alpha+\beta}}\right]$ for $\left|\frac{a-x}{a+c}\right|<1$, where, and $c$

$$
a \neq c
$$

being constants such that , then by Eqn. (2.2) there exists an integral representation

$$
\Psi_{a}^{(\alpha, \beta)}(s)=\frac{\Gamma(\beta)}{\Gamma(\alpha+\beta)} \frac{1}{(a \pm c)^{\alpha}} \int_{a}^{\infty}(x-a)^{\alpha+\beta-1}(x \pm c)^{-\beta} \varphi(x s) d x, \Re(s)>0 .
$$

Proof. In Eqn. (2.2), put $\psi(x)=\left[\frac{(x-a)^{\beta-1}}{(x \pm c)^{\alpha+\beta}}\right]$, for $\left|\frac{a-x}{a+c}\right|<1$, where, and being constants such that $a \neq c$, then, we evaluate (Mathai and Haubold 2008, p. 106, Exercise 2.4.6)

$$
{ }_{a} I_{x}^{\alpha}\left[\left[\frac{(x-a)^{\beta-1}}{(x \pm c)^{\alpha+\beta}}\right]\right]=\frac{\Gamma(\beta)}{\Gamma(\alpha+\beta)} \frac{1}{(a \pm c)^{\alpha}}(x-a)^{\alpha+\beta-1}(x \pm c)^{-\beta} .
$$

Finally, use (2.8) in (2.2), we obtain the representation (2.7). 
Corollary 2.3. Consider all conditions of the Theorem 2.2 and set $\varphi(x)=e^{-x}$ in it, then for $\alpha+\beta>0$, by representation (2.7) there exist a hypergeometric function

$$
\Psi_{a}^{(\alpha, \beta)}(s)=\frac{\Gamma(\beta) e^{-s a}}{[(a \pm c) s]^{\alpha+\beta}}{ }_{2} F_{0}\left(\beta, \alpha+\beta ;-;-\frac{1}{(a \pm c) s}\right), \Re(s)>0 .
$$

Proof. Consider the result (2.7) in the form

$\Psi_{a}^{(\alpha, \beta)}(s)=\frac{\Gamma(\beta)}{\Gamma(\alpha+\beta)} \frac{1}{(\alpha \pm c)^{\alpha}} \int_{0}^{\infty} x^{\alpha+\beta-1}(x+a \pm c)^{-\beta} \varphi((x+a) s) d x, \Re(s)>0$,

and $\operatorname{put} \varphi(x)=e^{-x}$, to get

$$
\Psi_{a}^{(\alpha, \beta)}(s)=\frac{\Gamma(\beta)}{\Gamma(\alpha+\beta)} \frac{e^{-s a}}{(a \pm c)^{\alpha}} \int_{0}^{\infty} e^{-s x} \chi^{\alpha+\beta-1}(x+a \pm c)^{-\beta} d x, \Re(s)>0
$$

$=\frac{\Gamma(\beta)}{\Gamma(\alpha+\beta)} \frac{e^{-s a}}{(a \pm c)^{\alpha+\beta}} \sum_{n=0}^{\infty} \frac{(\beta)_{n}}{n !}\left(-\frac{1}{a \pm c}\right)^{n} \int_{0}^{\infty} e^{-s x} x^{\alpha+\beta+n-1} d x$

$=\frac{\Gamma(\beta) e^{-s a}}{\{(a \pm c) s\}^{\alpha+\beta}} \sum_{n=0}^{\infty} \frac{(\beta)_{n}(\alpha+\beta)_{n}}{n !}\left(-\frac{1}{(a \pm c) s}\right)^{n}=\frac{\Gamma(\beta) e^{-s a}}{\{(a \pm c) s\}^{\alpha+\beta}}{ }_{2} F_{0}\left(\beta, \alpha+\beta ;-;-\frac{1}{(a \pm c) s}\right)$.

Further, on setting some particular manipulations in the result (2.9) as $\alpha=\frac{1}{2}, \beta=-\frac{n}{2},(a \pm c) s=z^{2}$, we may evaluate a classical relation in Hermite polynomials (Srivastava and Manocha 1984, p.40)

$$
\Psi_{a}^{\left(\frac{1}{2}-\frac{n}{2}\right)}\left(\frac{z^{2}}{(a+c)}\right)=\frac{\Gamma\left(-\frac{n}{2}\right) e^{-\frac{a}{(a \pm c)^{z^{2}}}}}{z}(2)^{-n} H_{n}(z) \forall n=1,2,3, \ldots
$$

Corollary 2.4. For all conditions of the Theorem 2.2, if in it set $\varphi(x s)={ }_{2} F_{1}\left(\alpha, \beta ; \delta ; \frac{x-a}{x+c} s\right)$, then for $\Re(s)>0, \alpha+\beta \neq 0, \pm 1, \pm 2, \ldots$, there exists a Gaussian hypergeometric function

$$
\Psi_{a}^{(\alpha, \beta)}(s)=\frac{(2 \pi)^{2}(-1)^{\beta+1}}{\Gamma(\alpha+\beta) \Gamma(1-\alpha-\beta) \Gamma(\alpha+1)}{ }_{2} F_{1}(\alpha, \alpha+\beta ; \delta ;(-s(a \pm c))) .
$$

Proof. For $\Re(s)>0$, in Eqn. (2.7) set $\varphi(x s)={ }_{2} F_{1}\left(\alpha, \beta ; \delta ; \frac{x}{x+a+c} s\right)$ to get

$$
\Psi_{a}^{(\alpha, \beta)}(s)=\frac{\Gamma(\beta)}{\Gamma(\alpha+\beta)} \frac{1}{(a \pm c)^{\alpha}} \int_{0}^{\infty} x^{\alpha+\beta-1}(x+a \pm c)^{-\beta}{ }_{2} F_{1}\left(\alpha, \beta ; \delta ;\left(\frac{x}{x+a \pm c}\right) s\right) d x
$$

$$
\begin{aligned}
& =\frac{\Gamma(\beta)}{\Gamma(\alpha+\beta)} \frac{1}{(a \pm c)^{\alpha}} \int_{0}^{\infty} x^{\alpha+\beta-1}(x-(\mp c-a))^{-\beta} F_{1}\left(\alpha, \beta ; \delta ;\left(\frac{x}{x-(\mp c-a)}\right) s\right) d x \\
& =\frac{\Gamma(\beta)}{\Gamma(\alpha+\beta)}(-1)^{\beta+1} \sum_{n=0}^{\infty} \frac{(\alpha)_{n}(\beta)_{n}(s(a \pm c))^{n}}{(\delta)_{n}} \int_{0}^{\infty}(-u)^{\alpha+\beta+n-1}(u-1)^{1-n-\beta-1} d x
\end{aligned}
$$


$=\frac{\Gamma(\beta)}{\Gamma(\alpha+\beta)}(-1)^{\beta+1} \sum_{n=0}^{\infty} \frac{(\alpha)_{n}(\beta)_{n}(s(\alpha \pm c))^{n}}{(\delta)_{n}} \int_{C}(-u)^{\alpha+\beta+n-1}(u-1)^{1-n-\beta-1} d u$

Now in Eqn. (2.13), use the formula due to Exton (1978), p. 74

$\int_{C}(-u)^{r-1}(u-1)^{r^{\prime}-1} d u=\frac{(2 \pi i)^{2}}{\Gamma(1-r) \Gamma\left(1-r^{\prime}\right) \Gamma\left(r+r^{\prime}\right)}$, we obtain

$\Psi_{a}^{(\alpha, \beta)}(s)=\frac{(2 \pi)^{2}}{\Gamma(1-\alpha-\beta) \Gamma(\alpha+\beta) \Gamma(\alpha+1)}(-1)^{\beta} \sum_{n=0}^{\infty} \frac{(\alpha)_{n}}{(1-\alpha-\beta)_{-n}(\delta)_{n}} \frac{(s(a \pm c))^{n}}{n !}$

$=\frac{(2 \pi)^{2}(-1)^{\beta+1}}{\Gamma(\alpha+\beta) \Gamma(1-\alpha-\beta) \Gamma(\alpha+1)}{ }_{2} F_{1}(\alpha, \alpha+\beta ; \delta ;(-s(a \pm c)))$ for $\alpha+\beta \neq 0, \pm 1, \pm 2, \ldots$.

Theorem 2.3. For $\Re(\alpha)>0, \Re(\beta)>0, \lambda \in \mathbb{C}, \Re(s)>0, a>0$, if $\psi(x)=\left[e^{\lambda x}(x-a)^{\beta-1}\right\rceil$, then by (2.2) there exists an integral representation

$$
\Psi_{a}^{(\alpha, \beta)}(\lambda, s)=\frac{\Gamma(\beta)}{\Gamma(\alpha+\beta)} e^{\lambda a} \int_{0}^{\infty} x^{\alpha+\beta-1}{ }_{1} F_{1}(\beta ; \alpha+\beta ; \lambda x) \varphi((x+a) s) d x .
$$

Proof. For $\Re(\beta)>0, \lambda \in \mathbb{C}$, set $\psi(x)=\left[e^{\lambda x}(x-a)^{\beta-1}\right\rceil$ in Eqn. (2.2), we write it in the form

$$
\Psi_{a}^{(\alpha, \beta)}(\lambda, s)=\int_{a}^{\infty} \varphi(x s)\left\{a_{a} I_{x}^{\alpha}\left[e^{\lambda x}(x-a)^{\beta-1}\right]\right\} d x, \Re(s)>0 .
$$

Now under the conditions $\Re(\alpha)>0, \Re(\beta)>0, \lambda \in \mathbb{C}$, the formula of $\left\{{ }_{a} I_{x}^{\alpha}\left[e^{\lambda x}(x-a)^{\beta-1}\right]\right\}$ is given by (Mathai and Haubold 2008, p. 106, Exercise 2.4.8)

$$
\left\{{ }_{a} I_{x}^{\alpha}\left[e^{\lambda x}(x-a)^{\beta-1}\right]\right\}=\frac{\Gamma(\beta)}{\Gamma(\alpha+\beta)} e^{\lambda a}(x-a)^{\alpha+\beta-1}{ }_{1} F_{1}(\beta ; \alpha+\beta ; \lambda(x-a)) .
$$

Thus apply the result (2.16) in Eqn. (2.15) and make some manipulations to obtain the result (2.14).

Corollary 2.5. If all conditions of the Theorem 2.3 are followed and set $\varphi(x)=e^{-x}$, there exists a formula

$$
\Psi_{a}^{(\alpha, \beta)}(\lambda, s)=\Gamma(\beta) e^{(\lambda-s) a} s^{-\alpha-\beta}\left(1-\frac{\lambda}{s}\right)^{-\beta}
$$

Proof. Consider the result (2.14) in which set $\varphi(x)=e^{-x}$, we get

$$
\Psi_{a}^{(\alpha, \beta)}(\lambda, s)=e^{(\lambda-s) a} \sum_{n=0}^{\infty} \frac{\Gamma(\beta)}{n ! \Gamma(\alpha+\beta)} \frac{(\beta)_{n} \lambda^{n}}{(\alpha+\beta)_{n}} \frac{1}{s^{\alpha+\beta+n}} \int_{0}^{\infty} e^{-u} u^{\alpha+\beta+n-1} d u
$$

$=\Gamma(\beta) \frac{e^{(\lambda-s) a}}{s^{\alpha+\beta}} \sum_{n=0}^{\infty} \frac{(\beta)_{n}}{n !} \frac{\lambda^{n}}{s^{n}}$.

By the result (2.18), we easily get (2.17). 
Again for further extensions, we define a Weyl fractional integral of the function $f(x)$ of order $\alpha$, denoted by ${ }_{x} W_{\infty}^{\alpha} f(x)$, for $\alpha \in \mathbb{C}$, in the form (Mathai and Haubold 2008, p. 103)

$$
{ }_{x} W_{\infty}^{\alpha} f(x)=\int_{x}^{\infty} \frac{(t-x)^{\alpha-1}}{\Gamma(\alpha)} f(t) d t, \Re(\alpha)>0,-\infty<x<\infty .
$$

Then, we present following theorem:

Theorem 2.4. For $\Re(\alpha)>0, a>0$, by Eqn. (2.2), there exists an integral representation involving Weyl integral function (2.19) as

$$
\Psi_{a}^{(\alpha)}(s)=\int_{0}^{\infty} \psi(x+a)\left\{{ }_{x} W_{\infty}^{\alpha} \varphi((x+a) s)\right\} d x, \Re(\alpha)>0, \Re(s)>0 .
$$

Proof. Use the basic property of the Weyl integral (2.19) due to (Mathai and Haubold 2008, p. 104) given by

(2.21) $\int_{0}^{\infty} \varphi(x)\left\{{ }_{0} I_{x}^{\alpha} \psi(x)\right\} d x=\int_{0}^{\infty} \psi(x)\left\{{ }_{x} W_{\infty}^{\alpha} \varphi(x)\right\} d x, \Re(\alpha)>0$,

in the result (2.2), we find (2.20).

Theorem 2.5. For $\Re(\alpha)>0, \lambda \in \mathbb{C}$, if $\psi(x)=\left\lceil e^{\lambda x}(x-a)^{\beta-1}\right\rceil$, then by the result (2.2), $\forall \Re(s)>0, a>0$, there exists an integral representation involving Weyl integral function

$$
\Psi_{a}^{(\alpha, \beta)}(\lambda, s)=e^{\lambda a} \int_{0}^{\infty} e^{\lambda x} x^{\beta-1}\left\{{ }_{x} W_{\infty}^{\alpha} \varphi((x+a) s)\right\} d x
$$

Proof. Introduce $\psi(x)=\left[e^{\lambda x}(x-a)^{\beta-1} \mid\right.$ in the Eqn. (2.2) and then make an appeal to the result (2.15) of the Theorem 2.3 in the form

$$
\Psi_{a}^{(\alpha, \beta)}(\lambda, s)=e^{\lambda a} \int_{0}^{\infty} \varphi((x+a) s)\left\{{ }_{0} I_{x}^{\alpha}\left[e^{\lambda x} x^{\beta-1}\right]\right\} d x
$$

Now, use the property (2.21) in the result (2.23), we find the representation (2.22).

Theorem 2.6. If

$$
\psi(x)=(x-a)^{\rho-1} H_{p, q}^{m, n}\left((x-a)^{\sigma} \mid \begin{array}{l}
\left(a_{p}, A_{p}\right) \\
\left(b_{q}, B_{q}\right)
\end{array}\right),{ }_{\text {where }} H_{p, q}^{m, n}\left((x-a)^{\sigma} \mid \begin{array}{l}
\left(a_{p}, A_{p}\right) \\
\left(b_{q}, B_{q}\right)
\end{array}\right), \text { is the }
$$

$H$-function, then for $\alpha \in \mathbb{C}, \Re(\alpha)>0, a_{i}, b_{j} \in \mathbb{C}, A_{i}, B_{j}>0, i=1,2, \ldots, p ; j=1,2, \ldots, q, \rho \in \mathbb{C}, \sigma>0$, and $\sigma \max _{1<i<n}\left[\frac{\Re\left(a_{i}\right)-1}{A_{i}}\right]+\Re(\rho)+\Re(\alpha)<1$, and $1+c \sigma>R(\rho)+\Re(\alpha)$, by the result (2.2), $\forall \Re(s)>0, a \geq 0$, there exists an integral representation

$$
\Psi_{a}^{(\alpha)}(s)=\int_{a}^{\infty}(x-a)^{\alpha+\rho-1} H_{p+1, q+1}^{m, n+1}\left((x-a)^{\sigma} \mid \begin{array}{c}
(1-\rho, \sigma),\left(a_{p}, A_{p}\right) \\
(1-\rho-\alpha, \sigma),\left(b_{q}, B_{q}\right)
\end{array}\right) \varphi(x s) d x .
$$


Proof. For $a \geq 0, \Re(\alpha)>0, \Re(s)>0$, introduce $\psi(x)=(x-a)^{\rho-1} H_{p, q}^{m, n}\left((x-a)^{\sigma} \mid \begin{array}{l}\left(a_{p}, A_{p}\right) \\ \left(b_{q}, B_{q}\right)\end{array}\right)$, in the result (2.2), we get

$$
\Psi_{a}^{(\alpha)}(s)=\int_{a}^{\infty} \varphi(x s)\left\{{ }_{a}^{\alpha}\left\{(x-a)^{\rho-1} H_{p, q}^{m, n}\left((x-a)^{\sigma} \mid \begin{array}{c}
\left(a_{p}, A_{p}\right) \\
\left(b_{q}, B_{q}\right)
\end{array}\right)\right\}(x)\right\} d x .
$$

Again, in the integrand of the integral (2.25) define the $H$-function (Mathai et. al 2010, p.2-3)

$H_{p, q}^{m, n}\left(z \mid \begin{array}{l}\left(a_{p}, A_{p}\right) \\ \left(b_{q}, B_{q}\right)\end{array}\right)=\frac{1}{2 \pi i} \int_{c-i \infty}^{c+i \infty} \Theta(s) z^{-s} d s \quad, \quad$ where, $\quad \Theta(s)=\frac{\prod_{j=1}^{m} \Gamma\left(b_{j}+B_{j} s\right) \prod_{l=1}^{n} \Gamma\left(1-a_{l}-A_{l} s\right)}{\prod_{j=m+1}^{q} \Gamma\left(1-b_{j}-B_{j} s\right) \prod_{l=n+1}^{p} \Gamma\left(a_{l}+A_{l} s\right)} \quad$ and , $i=\sqrt{(-1)}$, the poles of $\Gamma\left(b_{j}+B_{j} s\right), j=1, \ldots, m$ are separated from those of $\Gamma\left(1-a_{l}-A_{l} s\right), l=1,2, \ldots n$. The integral converges for $z \neq 0$, and $\sum_{l=1}^{n} A_{l}-\sum_{l=n+1}^{p} A_{l}+\sum_{j=1}^{m} B_{j}-\sum_{j=m+1}^{q} B_{j}>0$, then, we find

$$
{ }_{a} I_{x}^{\alpha}\left\{(x-a)^{\rho-1} H_{p, q}^{m, n}\left((x-a)^{\sigma} \mid \begin{array}{l}
\left(a_{p}, A_{p}\right) \\
\left(b_{q}, B_{q}\right)
\end{array}\right)\right\}(x)
$$

$=\frac{1}{\Gamma(\alpha)} \int_{a}^{x}(x-t)^{\alpha-1}(t-a)^{\rho-1} H_{p, q}^{m, n}\left((t-a)^{\sigma} \mid \begin{array}{l}\left(a_{p}, A_{p}\right) \\ \left(b_{q}, B_{q}\right)\end{array}\right) d t$

$=\frac{1}{\Gamma(\alpha)} \int_{c-i \infty}^{c+i \infty} \Theta(s) \int_{a}^{x}(x-t)^{\alpha-1}(t-a)^{\rho-\sigma s-1} d t d s$

now put $(t-a)=u(x-a)$, in the inner integral we get

$=(x-a)^{\alpha+\rho-1} \int_{c-i \infty}^{c+i \infty} \Theta(s) \frac{\Gamma(\rho-\sigma s)}{\Gamma(\alpha+\rho-\sigma s)}(x-a)^{-\sigma s} d s$

as on appealing the definition of $\mathrm{H}$-function given in Eqn. (2.25), it becomes

$=(x-a)^{\alpha+\rho-1} H_{p+1, q+1}^{m, n+1}\left((x-a)^{\sigma} \mid \begin{array}{c}(1-\rho, \sigma),\left(a_{p}, A_{p}\right) \\ (1-\rho-\alpha, \sigma),\left(b_{q}, B_{q}\right)\end{array}\right)$,

provided that $\alpha \in \mathbb{C}, \Re(\alpha)>0, a_{l}, b_{j} \in \mathbb{C}, A_{l}, B_{j}>0, l=1,2, \ldots, p ; j=1,2, \ldots, q, \rho \in \mathbb{C}, \sigma>0$, and it satisfy

$\sigma \max _{1<i<n}\left[\frac{\Re\left(a_{i}\right)-1}{A_{i}}\right]+\Re(\rho)+\Re(\alpha)<1$ and $1+c \sigma>R(\rho)+\Re(\alpha)$.

Hence the results (2.25) and (2.26) under the given conditions in the Theorem 2.6, give us the integral representation (2.24). 
Further in the integral (2.24) on specializing various values of the test function ${ }^{\phi}$, we derive many functions. Due to lack of space, we omit them.

3. The functions of contour integral representations

In this section, by our obtained results given in Section $\mathbf{2}$ we derive some of contour integral representations existing in the class $\Omega_{a}^{(\alpha)}$.

Theorem 3.1. For $\Re(\alpha)>0, \quad \min \{\Re(\xi), \Re(\alpha+\beta), z\}>0, \quad \lambda \in \mathbb{C}$, such that $|\lambda|<\frac{\pi}{2}$, if $\psi(x)=\left[e^{\lambda x}(x-a)^{\beta-1}\right\rceil$, then $\forall \Re(s)>0, a>0$, in the complex $\xi$ - plane by the result (2.2), there exists following contour integral representation as

$$
\Psi_{a}^{(\alpha)}(\lambda, s)=\Gamma(\beta) e^{\lambda a} \frac{1}{2 \pi i} \int_{c-i \infty}^{c+i \infty} e^{\lambda \xi} \xi^{-\alpha-\beta} F(\xi, s) d \xi \text {, where, } c>R(\xi)
$$

and

$$
F(\xi, s)=\int_{0}^{\infty}\left(1-\frac{z}{\xi}\right)^{-\beta} \varphi((z+a) s) d z
$$

Proof. Use $\psi(x)=\left[e^{\lambda x}(x-a)^{\beta-1}\right\rceil$ in the Eqn. (2.2) and thus consider the Eqn. (2.14) of the Theorem 2.3 in the form

$$
\Psi_{a}^{(\alpha)}(\lambda, s)=\Gamma(\beta) e^{\lambda a} \int_{0}^{\infty} \frac{z^{\alpha+\beta-1}}{\Gamma(\alpha+\beta)}{ }_{1} F_{1}(\beta ; \alpha+\beta ; \lambda z) \varphi((z+a) s) d z
$$

Now in right hand side of (3.3), for $c>R(\xi), \Re(\alpha)>0, \min \{\Re(\xi), \Re(\alpha+\beta), z\}>0,|\lambda|<\frac{\pi}{2}$, use the formula (Chandel and Kumar 2020, p.233, Eqn. (1.4), Fejzullahu 2016, Kumar 2020, p. 85, Eqn. (2.1)) as

$$
\begin{aligned}
& \frac{z^{\alpha+\beta-1}}{\Gamma(\alpha+\beta)}{ }_{1} F_{1}(\beta ; \alpha+\beta ; \lambda z)=\frac{1}{2 \pi i} \int_{c-i \infty}^{c+i \infty} e^{\lambda \xi} \xi^{-\alpha-\beta}\left(1-\frac{z}{\xi}\right)^{-\beta} d \xi \\
& \Psi_{a}^{(\alpha)}(\lambda, s)=\Gamma(\beta) e^{\lambda a} \frac{1}{2 \pi i} \int_{c-i \infty}^{c+i \infty} e^{\lambda \xi} \xi^{-\alpha-\beta}\left\{\int_{0}^{\infty}\left(1-\frac{z}{\xi}\right)^{-\beta} \varphi((z+a) s) d z\right\} d \xi
\end{aligned}
$$

Again, in the inner integral of (3.5) use the definition (3.2), we obtain (3.1).

Remark 3.1. In Eqn. (2.2), on setting $\psi(x)=(x-a)^{\sigma-1}$ and putting $a=y$, and to get

$$
y_{x}^{\alpha}\left\{(x-y)^{\sigma-1}\right\}=\frac{\Gamma(\sigma)}{\Gamma(\sigma+\alpha)}(x-y)^{\alpha+\sigma-1}=\frac{(x-y)^{\alpha+\sigma-1}}{(\sigma)_{\alpha}} .
$$


Hence, for $\sigma=1, x>y>0$, we get $I_{y}^{\alpha}\left\{(x-y)^{\sigma-1}\right\}=\frac{(x-y)^{\alpha}}{\Gamma(1+\alpha)}$ and then by Eqn. (3.6), we may find an interesting relation with Heaviside type function for $\alpha=k-1, \sigma=1$, as

$$
\left.{ }_{y} I_{x}^{\alpha}\left\{(x-y)^{\sigma-1}\right\}\right|_{\sigma=1, \alpha=k-1}=\frac{(x-y)^{k-1}}{\Gamma(k)}
$$

$=H^{(k)}(x-y)=\left\{\begin{array}{c}\frac{(x-y)^{k-1}}{\Gamma(k)} ; x>y>0, k \geq 1, \\ 0 ; x<y<0 \text { or } x=y, k \geq 2 . \\ \frac{1}{2} ; x=y, k=1 .\end{array}\right.$

Here, $H^{(k)}(x)$ is the k- th Heaviside function given by (Lapidus and Frankenhuijsen 2006, p. 139, Eqn. (5.10))

$$
H^{(k)}(x)=\left\{\begin{array}{c}
\frac{x^{k-1}}{\Gamma(k)} ; x>0, k \geq 1, \\
0 ; x<0 \text { or } x=0, k \geq 2, \\
\frac{1}{2} ; x=0, k=1 .
\end{array}\right.
$$

In respect of the Remark 3.1, further we extend our work as on setting $a=y, \psi(x)=(x-y)^{\sigma-1}$ in Eqn. (2.2), to get it in the form

$$
\Psi_{y}^{(\alpha, \sigma)}(s)=\Gamma(\sigma) \int_{y}^{\infty} \varphi(x s) \frac{(x-y)^{\alpha+\sigma-1}}{\Gamma(\sigma+\alpha)} d x, \Re(\alpha)>0, \Re(s)>0 .
$$

Particularly, by the Eqn. (3.8) we find a classical relation

$$
\Psi_{y}^{(k-1,1)}(s)=\int_{y}^{\infty} \varphi(x s) H^{(k)}(x-y) d x, \Re(s)>0 .
$$

Again, on integrating the formula (3.8) with respect to y, from $y=0_{\text {to }} y=\infty$, and as on using the techniques of the order of integration, given in the Eqn. (1.4), we find an important result

$$
\int_{0}^{\infty} \Psi_{y}^{(\alpha, \sigma)}(s) d y=\Gamma(\sigma) \int_{0}^{\infty} \int_{y}^{\infty} \varphi(x s) \frac{(x-y)^{\alpha+\sigma-1}}{\Gamma(\sigma+\alpha)} d x d y=\Gamma(\sigma) \int_{0}^{\infty} \varphi(x s)\left\{\int_{0}^{x} \frac{(x-y)^{\alpha+\sigma-1}}{\Gamma(\sigma+\alpha)} d y\right\} d x
$$

$$
\begin{aligned}
& =\Gamma(\sigma) \int_{0}^{\infty} \varphi(x s)\left(I_{0}^{\alpha+\sigma-1}\{1\}\right) d x=\frac{\Gamma(\sigma)}{\Gamma(\sigma+\alpha+1)} \int_{0}^{\infty} \varphi(x s) x^{\alpha+\sigma+1-1} d x \\
& =\frac{\Gamma(\sigma)}{\Gamma(\sigma+\alpha+1)} \frac{1}{s^{\alpha+\sigma+1}} \int_{0}^{\infty} \varphi(x) x^{\alpha+\sigma+1-1} d x \\
& =\frac{\Gamma(\sigma)}{\Gamma(\sigma+\alpha+1)} \frac{G(\alpha+\sigma+1)}{s^{\alpha+\sigma+1}}=\frac{\Gamma(\sigma)}{\Gamma(\lambda)} \frac{G(\lambda)}{s^{\lambda}},
\end{aligned}
$$

where, for $\alpha+\sigma+1=\lambda$, we consider that 
(3.11) $G(\lambda)=\int_{0}^{\infty} x^{\lambda-1} \varphi(x) d x$.

So that by the Eqn. (3.11), we get a contour integral representation (see also Exton 1978 p. 111)

$$
\varphi(x)=\frac{1}{2 \pi i} \int_{c-i \infty}^{c+i \infty} x^{-\lambda} G(\lambda) d \lambda
$$

Then from (3.12), we write

$$
\varphi(y)=\frac{1}{2 \pi i} \int_{c-i \infty}^{c+i \infty} y^{-\lambda} G(\lambda) d \lambda
$$

Thus on using (3.11) and (3.13), for $\lambda=\alpha+\sigma+1$, we get

$$
\varphi(y)=\frac{1}{2 \pi i} \int_{c-i \infty}^{c+i \infty} y^{-\lambda} \int_{0}^{\infty} x^{\lambda-1} \varphi(x) d x d \lambda=\int_{0}^{\infty}\left\{\frac{1}{2 \pi i} \int_{c-i \infty}^{c+i \infty} y^{-\lambda} x^{\lambda-1} d \lambda\right\} \varphi(x) d x
$$

The Eqn. (3.14) claims an equality if we define a Dirac delta function in the complex plane

$$
\delta(x-y)=\frac{1}{2 \pi i} \int_{c-i \infty}^{c+i \infty} y^{-\lambda} x^{\lambda-1} d \lambda, \text { where, } \lambda=\alpha+\sigma+1 .
$$

It is noted that in other notation of the Dirac delta function, it is introduced in (Lapidus and Frankenhuijsen 2006, p. 135).

Then, the Eqns. (3.14) and (3.15) give the equality

$$
\varphi(y)=\int_{0}^{\infty} \varphi(x) \delta(x-y) d x
$$

Ultimately, on application of the Eqns. (3.10) and (3.12), we obtain a contour integral representation in the form

$$
\varphi(x)=\frac{1}{2 \pi i} \int_{c-i \infty}^{c+i \infty} x^{-\lambda} \mathrm{s}^{\lambda} \frac{\Gamma(\lambda)}{\Gamma(\lambda-\alpha-1)} P(\alpha, \lambda-\alpha-1, s) d \lambda
$$

Here, we consider that $P(\alpha, \lambda-\alpha-1, s)=\int_{0}^{\infty} \Psi_{y}^{(\alpha, \lambda-\alpha-1)}(s) d y$.

\section{Certain boundary value problems}

In this section to propose our investigation, we make an appeal to the general theory for finding out integral representations due to (Hochstadt 1986, p.100) and by reverse theory we derive certain value problems via following theorems and examples.

Theorem 4.1. If there exist an integral representation

(4.1) $Y(x)=\int_{a}^{b} e^{x t} f(t) d t, b>a$,

provided that 
(4.2) $\Delta_{t} f(t)=0$ and $\left.\left\{e^{x t} f(t)\right\}\right|_{t=a} ^{t=b}=0, \Delta_{t}$ is any differential operator with respect to $t$.

Then there exists a differential equation

(4.3) $\nabla_{x} Y(x)=\Delta_{t}^{*} \int_{a}^{b} e^{x t} f(t) d t=0$, where, $\Delta_{t}^{*}$ is the adjoint operator of the operator $\Delta_{t^{*}}$

Proof. By the equation (4.3), we find that

(4.4) $\nabla_{x} Y(x)=\Delta_{t}^{*} \int_{a}^{b} e^{x t} f(t) d t=\int_{a}^{b} e^{x t} \Delta_{t}^{*} f(t) d t+\int_{a}^{b} f(t) \Delta_{t}^{*} e^{x t} d t$

$=\int_{a}^{b} e^{x t} \Delta_{t}^{*} f(t) d t+\int_{a}^{b}\left[e^{x t} \Delta_{t} f(t)-\frac{d}{d t}\left\{e^{x t} f(t)\right\}\right] d t=0$

(Since, $\left.f(t) \Delta_{t} e^{x t}-e^{x t} \Delta_{t}^{*} f(t)=\frac{d}{d t}\left\{e^{x t} f(t)\right\}\right)$. (See the identity due to Hochstadt (1986, p. 101, Eqn.

In the equation (4.4), the equality holds if and only if

(4.5) $\Delta_{t}^{*} f(t)=\Delta_{t} f(t)=0$ and $\left.\left\{e^{x t} f(t)\right\}\right|_{t=a} ^{t=b}=0$.

Finally, on making an appeal to (4.4) and (4.5), we get differential equation (4.3), if the conditions (4.5) are satisfied.

Hence, the Theorem 4.1 holds good.

Corollary 4.1. If all conditions of Theorem 2.5 are followed and if in it the equivalence relation $\Delta_{x}^{*} \equiv \nabla_{\lambda}$ is followed, where, $\Delta_{\lambda}^{*}$ is the adjoint operator of the operator $\Delta_{\lambda}$. Again here, following equation and the boundary conditions hold

$$
\Delta_{x}\left[x^{\beta-1}\left\{{ }_{x} W_{\infty}^{\alpha} \varphi((x+a) s)\right\}\right]=0 \text { and }\left.\left[e^{\lambda x} x^{\beta-1}\left\{{ }_{x} W_{\infty}^{\alpha} \varphi((x+a) s)\right\}\right]\right|_{x \rightarrow \infty} ^{x \rightarrow \infty}=0 .
$$

Then there, a differential equation also holds

(4.7) $\nabla_{\lambda} \Psi_{a}^{(\alpha)}(\lambda)=0$.

Proof. Take the operator $\Delta_{x}^{*}$, an adjoint of the operator $\Delta_{x}$ and then, by the Equation (4.6), we have $\Delta_{x}^{*}\left[x^{\beta-1}\left\{{ }_{x} W_{\infty}^{\alpha} \varphi((x+a) s)\right\}\right]=0$.

Now, by the operator relation $\Delta_{x}^{*} \equiv \nabla_{\lambda}$ we find $\nabla_{\lambda}$ by the equation

$\Delta_{x}^{*} e^{\lambda x}=\nabla_{\lambda} e^{\lambda x}$, then by the Theorem 2.5, and using the Theorem 4.1, we get the operator $\nabla_{\lambda} \cdot$ Now, then under the conditions of (4.6), it satisfies the equation

$\nabla_{\lambda} \Psi_{a}^{(\alpha)}(\lambda)=\Delta_{x}^{*} \int_{0}^{\infty} e^{\lambda x} x^{\beta-1}\left\{{ }_{x} W_{\infty}^{\alpha} \varphi((x+a) s)\right\} d x=0$.

Example 4.1. If all conditions of Theorem 2.5 are followed and again, following equation and boundary conditions are satisfied

(4.8) $\left(-\frac{d}{d x}+x^{2}\right)\left[x^{\beta-1}\left\{{ }_{x} W_{\infty}^{\alpha} \varphi((x+a) s)\right\}\right]=0$ and $\left.\left[e^{\lambda x} x^{\beta-1}\left\{{ }_{x} W_{\infty}^{\alpha} \varphi((x+a) s)\right\}\right]\right|_{x \rightarrow \infty} ^{x \rightarrow \infty}=0$.

Then, a differential equation holds in the form

$$
\left(\frac{d^{2}}{d \lambda^{2}}+\lambda\right) \Psi_{a}^{(\alpha)}(\lambda)=0 \text {. }
$$


Solution. Here in the Example 4.1, $\Delta_{x}=\left(-\frac{d}{d x}+x^{2}\right)$ so that its adjoint operator is found by $\Delta_{x}^{*}=\left(\frac{d}{d x}+x^{2}\right)$

and thus

$$
\Delta_{x}^{*} e^{\lambda x}=\left(\frac{d}{d x}+x^{2}\right) e^{\lambda x}=\left(\lambda+x^{2}\right) e^{\lambda x}=\left(\lambda+\frac{d^{2}}{d \lambda^{2}}\right) e^{\lambda x}=\nabla_{\lambda} e^{\lambda x} .
$$

Hence by the Corollary 4.1, there follows the equation

$\left(\frac{d^{2}}{d \lambda^{2}}+\lambda\right) \Psi_{a}^{(\alpha)}(\lambda)=0$.

Example 4.2. If all conditions of Theorem 2.5 are followed and again, following equation and boundary conditions are satisfied

$$
\left(x^{\beta+1}+3 x^{\beta}+2 x^{\beta-1}\right)\left\{{ }_{x} W_{\infty}^{\alpha} \varphi((x+a) s)\right\}=0
$$

and

$$
\left.\left[e^{\lambda x} x^{\beta-1}\left\{{ }_{x} W_{\infty}^{\alpha} \varphi((x+a) s)\right\}\right]\right|_{x \rightarrow \infty} ^{x \rightarrow \infty}=0 .
$$

Then, following differential equation holds

$$
\left(\frac{d^{2}}{d \lambda^{2}}+3 \frac{d}{d \lambda}+2\right) \Psi_{a}^{(\alpha)}(\lambda)=0 \text {. }
$$

Solution. Consider the equation (4.8) and write that in the form

(4.10) $\left(x^{2}+3 x+2\right)\left[x^{\beta-1}\left\{{ }_{x} W_{\infty}^{\alpha} \varphi((x+a) s)\right\}\right]$, by which we have $\Delta_{x}=\left(x^{2}+3 x+2\right)$ so that its adjoint operator is found by

$\Delta_{x}^{*}=\left(x^{2}+3 x+2\right)$ and thus $\Delta_{x}^{*} e^{\lambda x}=\left(x^{2}+3 x+2\right) e^{\lambda x}=\left(\frac{d^{2}}{d \lambda^{2}}+3 \frac{d}{d \lambda}+2\right) e^{\lambda x}=\nabla_{\lambda} e^{\lambda x}$.

Hence by the Corollary 4.1, there follows the equation $\left(\frac{d^{2}}{d \lambda^{2}}+3 \frac{d}{d \lambda}+2\right) \Psi_{a}^{(\alpha)}(\lambda)=0$.

In same manner of the Corollary 4.1, by the Theorem 3.1 following equation hold:

Corollary 4.2. If all conditions of Theorem 3.1 are followed and if in it the equivalence relation $\Delta_{\xi}^{*} \equiv \nabla_{\lambda}$ is followed, where, $\Delta \xi$ is the adjoint operator of the operator $\Delta_{\xi}$. Again here, following equation and the boundary conditions are satisfied

$$
\Delta_{\xi}\left[\xi^{-\alpha-\beta} F(\xi, s)\right]=0 \text { and }\left[e^{\lambda \xi} \xi^{-\alpha-\beta} F(\xi, s)\right] \mid \begin{aligned}
& \xi \rightarrow c+i \infty \\
& \xi \rightarrow c-i \infty
\end{aligned}=0 .
$$

Then there, a differential equation also holds

$$
\nabla_{\lambda} \Psi_{a}^{(\alpha)}(\lambda, s)=0 \text {. }
$$

\section{Conclusion}

This research work is interdisciplinary approach of fractional calculus, fractal geometry, integral representations, complex analysis, differential operators and mathematical physics. The researchers concerning these fields may extend their researches by reading this paper. In Section 1, we define a generalized class (1.8) of a double 
computer programs; John Willey and Sons, New York.

Fejzullahu BXh (2016) On the integral representations for confluent hypergeometric function, Proc. R. Soc. A. 472: $1-8$.

functions and and thus these integral representations are the members to a class $\Omega_{a}^{(\alpha)}$, given in (1.8). In the Section 3, we obtain contour integral representation on using our some of obtained results of Section 2. Chandel and Kumar (2020) have recently made the application of contour integral representation in solving of the initial value problems consisting of Riemann -Liouville operators. Fejzullahu (2016) has obtained various contour integral representations of the special functions. Thus by this our work, on setting particular values of the testing function $\varphi$, readers may do more extensions in these researches. Finally in Section 4, we obtain certain boundary value problems via the integral representations obtained in previous sections deriving some results reversing to Hochstadt (1986, p.100). This work is of quit interesting and consisting of interdisciplinary perspective of many areas of Mathematics and Physical Sciences.

\section{References}

Chandel RCS and Kumar H (2020) Contour integral representations of two variables generalized hypergeometric function of Srivastava and Daoust with their applications to initial value problems of arbitrary order, J $\widetilde{n} \bar{a}_{n} \bar{a}_{\text {bha }} \mathbf{5 0}$ (1): 232 242.

Diethelm K (2010) The Analysis of Fractional Differential Equations; An applicationoriented exposition using differential operators of Caputo type; Springer, New York.

Exton H (1978) Handbook of Hypergeometric Integrals; Theory, applications, tables and
Hochstadt H (1986) The Functions of Mathematical Physics, Dover Publications Inc., New York.

Kumar H (2020) Application in initial value problems via operational techniques on a contour integral for Srivastava - Daoust function of two variables, $\tilde{m} \bar{a}_{n} \bar{a}_{b h a} \mathbf{5 0}$ (2): $84-92$.

Lapidus ML and Frankenhuijsen Mvan (2000) Fractal Geometry and Number Theory; Complex dimensions of fractal strings and zeros of zeta functions; Birkh $\ddot{a}_{\mathrm{user}}$, Boston.

Lapidus ML and Frankenhuijsen M. van (2006) Fractal Geometry, Complex Dimensions and Zeta Functions; Geometry and spectra of fractal strings; Springer, New York.

Mathai AM and Haubold HJ (2008) Special Functions for Applied Scientists, Springer, New York. Mathai AM, Saxena RK and Haubold HJ (2010) The H-Function; Theory and Application; Springer, New York.

Oldham KB and Spanier J (2002) The Fractional Calculus;Theory and application of differentiation and integration to arbitrary order; Dover Publications Inc., New York.

Srivastava HM and Manocha HL (1984) A Treatise on Generating Functions, John Willey and Sons, New York. 